\title{
Early-onset inflammatory bowel disease caused by mutant IL10 receptor
}

\author{
E O Glocker ${ }^{1 \dagger}$, D Kotlarz ${ }^{2 \dagger}$, K Boztug $^{2 \dagger}$, E M Gertz ${ }^{3}$, A A Schäffer ${ }^{3}$, F Noyan ${ }^{2}$, M Perro ${ }^{1}$, J Diestelhorst ${ }^{2}$, A Allroth ${ }^{2}$, \\ D Murugan ${ }^{2}$, N Hätscher ${ }^{2}$, D Pfeifer ${ }^{4}$, K W Sykora ${ }^{2}$, M Sauer $^{2}$, H Kreipe $^{5}$, M Lacher $^{6}$, R Nustede $^{7}$, C Woellner ${ }^{1}$,

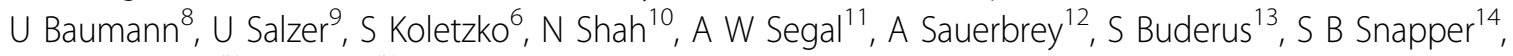 \\ B Grimbacher ${ }^{1 *+}$, C Klein ${ }^{2^{*+}}$
}

From 5th European Workshop on Immune-Mediated Inflammatory Diseases

Sitges-Barcelona, Spain. 1-3 December 2010

\section{Background}

The molecular etiology of inflammatory bowel diseases (IBD) is largely unknown.

\section{Methods}

We performed genetic linkage analysis and candidate gene sequencing in two unrelated consanguineous families with children affected by early-onset IBD. We screened six additional patients for mutations in two candidate genes and carried out functional assays in patients' peripheral blood mononuclear cells. We treated one patient with an allogeneic hematopoietic stem cell transplant (HSCT).

\section{Results}

We identified three distinct homozygous mutations in the genes IL1ORA and IL1ORB, encoding the IL10R1 and IL10R2 proteins, respectively (which form a heteromer to make up the interleukin-10 receptor) in four of nine patients with early-onset colitis. The mutations abrogate IL10-induced signaling, as demonstrated by deficient STAT3 phosphorylation upon IL10 stimulation. Consistent with this observation is the increased secretion of $\mathrm{TNF} \alpha$ and other proinflammatory cytokines from peripheral blood mononuclear cells from IL10R-deficient patients, suggesting that IL10-dependent "negative feedback" regulation is disrupted in these cells. One patient was successfully treated by an allogeneic HSCT.

\section{Conclusions}

Mutations in genes encoding the IL10R subunit proteins cause human enterocolitis, involving hyperinflammatory immune responses in the intestine. Allogeneic HSCT may offer a cure for IL10 receptor deficiency.

\begin{abstract}
Author details
${ }^{1}$ Dept. of Immunology, Royal Free Hospital and University College London, London, UK. ²Dept. of Pediatric Hematology/Oncology, Hannover Medical School, Hannover, Germany. ${ }^{3}$ National Center for Biotechnology Information, $\mathrm{NIH}, \mathrm{DHHS}$, Bethesda, MD, USA. ${ }^{4}$ Dept. of Hematology/Oncology, Core Facility II Genomics, Freiburg University Medical Center, Freiburg, Germany. ${ }^{5}$ Dept. of Pathology, Hannover Medical School, Hannover, Germany. ${ }^{6}$ Dr. von Hauner'sches Kinderspital, Ludwig-Maximilian University, Munich, Germany. ${ }^{7}$ Dept. of Pediatric Surgery, Hannover Medical School, Hannover, Germany. ${ }^{8}$ Dept. of Pediatric Pulmonology, Hannover Medical School, Hannover, Germany. ${ }^{9}$ Dept. of Rheumatology and Clinical Immunology, University Hospital Freiburg, Freiburg, Germany. ${ }^{10}$ Dept. of Paediatric Gastroenterology, Great Ormond Street Hospital, University College London, London, UK. ${ }^{11}$ Dept. of Medicine, University College London, London, UK. ${ }^{12}$ Dept. of Pediatrics, HELIOS Hospital Erfurt, Erfurt, Germany. ${ }^{13}$ Dept. of Pediatrics, St.-Marien-Hospital Bonn, Bonn, Germany. ${ }^{14}$ Massachusetts General Hospital, Harvard Medical School, Boston MA, USA.
\end{abstract}

Published: 25 November 2010

doi:10.1186/1479-5876-8-S1-I12

Cite this article as: Glocker et al.: Early-onset inflammatory bowel

disease caused by mutant IL10 receptor. Journal of Translational Medicine 2010 8(Suppl 1):112.

\footnotetext{
† Contributed equally

'Dept. of Immunology, Royal Free Hospital and University College London, London, UK

Dept. of Pediatric Hematology/Oncology, Hannover Medical School,

Hannover, Germany

Full list of author information is available at the end of the article
} 